\title{
Uso de la moxidectina para el tratamiento de los parásitos internos y externos de los animales.
}

Revisión

Gabriela Aguilar-Tipacamú, Roger I. Rodríguez-Vivas.

Departamento de Parasitología, Facultad de Medicina Veterinaria y Zootecnia, Universidad Autónoma de Yucatán. Mérida, Yucatán, México.

\section{RESUMEN.}

El objetivo de la presente revisión fue realizar una búsqueda de información actualizada de la moxidectina, donde se describe su composición química, farmacodinamia, seguridad, persistencia, dosificación y forma de presentación. Así mismo, se reporta el uso de la moxidectina para el tratamiento de parásitos internos y externos de los ovinos, caprinos, equinos, bovinos, porcinos, caninos, venados y ciervos rojos, en experimentos realizados por diversos autores. Se concluye que la moxidectina es altamente efectiva para el tratamiento de nemátodos y artrópodos de los animales. (Rev Biomed 2002; 13:43-51)

Palabras clave: Moxidectina, nemátodos, artrópodos, patología animal.

\section{SUMARY.}

Use of moxidectin in the treatment of internal and external parasites in animals.

The aim of the present review was to present up-to-date information of moxidectin. Its chemical composition, pharmacodinamics, safety, persistance, dosification and type of presentation is described. The use of moxidectin to treat internal and external parasites in sheep, goats, equine, cattle, swine, dogs, deer and red deer are also reported, in studies carried out by different authors. It is concluded that moxidectin is highly effective in the treatment of nematodes and arthropods in animals. (Rev Biomed 2002; 13:43-51)

Key Words: Moxidectin, nematodes, arthropodes, animal pathology.

Solicitud de sobretiros: MSc. Roger I. Rodríguez-Vivas. Departamento de Parasitología, Facultad de Medicina Veterinaria y Zootecnia, Universidad Autónoma de Yucatán. Apdo. Postal 4-116 Itzimná. Mérida, Yucatán, México. Fax: (999) 94232 05. E-mail: rvivas@tunku.uady.mx 


\section{G Aguilar-Tipacamú, RI Rodríguez-Vivas.}

\section{INTRODUCCIÓN.}

La moxidectina es una lactona macrocíclica que se sintetizó en 1990 y ha demostrado tener un amplio espectro contra parásitos internos y externos de los animales. Es un fármaco seguro que puede usarse varias veces la dosis terapéutica sin presentar cuadros clínicos de intoxicación en la mayoría de los animales domésticos $(1,2)$.

La moxidectina incrementa la liberación del ácido gammaaminobutírico (GABA) de los sinaptosomas del sistema nervioso; sin embargo, se asume que tiene otro mecanismo de acción desconocido $(2,3)$.

Las dosis terapéuticas usadas son de 0.2 a 1.5 $\mathrm{mg} / \mathrm{kg}$ de peso vivo (p.v.), con resultados satisfactorios. Tiene efecto contra las fases adultas e inmaduras de nemátodos y artrópodos $(4,5)$.

La moxidectina interfiere en la reproducción de parásitos a través de un mecanismo desconocido, causando reducción en la oviposición en garrapatas, formación de huevos anormales en nemátodos de rumiantes y esterilidad de machos y hembras del género Dirofilaria (1).

Las presentaciones de este producto son al $0.1 \%$ y $0.2 \%$ en suspensión oral, al $1.0 \%$ en solución inyectable y al $0.5 \%$ en solución de derrame (“pour-on”) (1).

\section{COMPOSICIÓN.}

Es una nueva lactona macrocíclica semisintética, producida por modificación química de la nemadectina; el principal componente de la LL-F28249 producida por fermentación de Streptomyces cyanogriseus noncyanogenus. $\mathrm{Su}$ estructura química se relaciona con la milbemicina y avermectinas, con las que comparte no sólo la similitud de su molécula sino además el tipo de absorción y farmacocinética (2).

\section{FARMACODINAMIA.}

Las avermectinas inhiben la motilidad e incrementan aparentemente la liberación del GABA de los sinaptosomas del sistema nervioso. El GABA es un neurotransmisor inhibitorio de los estímulos nerviosos en la placa neuromuscular. El aumento de la liberación del GABA incrementa (hiperpolariza) el potencial normal en reposo de las células postsinápticas, haciendo más difícil la neurotransmisión de los estímulos a los músculos; por ello, las fibras musculares no se contraen bajo la influencia de la avermectina, los vermes se paralizan y son eliminados (6).

Mudd y Parker (7), mencionan que en los nemátodos, la moxidectina abre los canales de cloruro en la conexión postsináptica, y permite el flujo de iones, produciendo un estado irreversible de descanso, parálisis y muerte del parásito.

La moxidectina al igual que los fármacos del mismo grupo, es un agonista del GABA, pero aún falta mucho por aclarar debido a que este medicamento presenta efecto contra parásitos resistentes a otras ivermectinas, lo que sugiere un diferente y desconocido mecanismo de acción $(2,3)$.

Gill y Lacey (8), mencionan que las lactonas macrocíclicas tienen dos efectos en los nemátodos gastrointestinales; el primero causan parálisis de la musculatura reduciendo con ello la motilidad, y el segundo causan parálisis de la faringe del gusano provocando un estado de inanición que declinan el almacenamiento de energía.

La falta de eficacia de la moxidectina contra céstodos y tremátodos, está ligada a la ausencia de requerimientos del GABA para las funciones metabólicas (2).

La moxidectina es más lipofílica e hidrofóbica que la ivermectina. Se absorbe por todas las vías debido a que es muy liposoluble, se distribuye ampliamente en los tejidos y se acumula sobre todo en la luz intestinal por su ciclo biliar, en grasa y en piel; lo que permite ser usada como acaricida con excelentes resultados. La vida media en bovinos es de nueve a 11 días en promedio, con un efecto residual de tres semanas (2).

En cabras es rápidamente absorbida y alcanza una máxima concentración en sangre a los $0.366 \pm$ 0.03 días después de la administración oral y 0.396 
Tratamiento con moxidectina en animales.

\pm 0.05 días al ser inyectada subcutáneamente. El promedio de tiempo residual de la moxidectina oral y subcutánea es de $10.29 \pm 0.83$ y $12.43 \pm$ 1.28 días respectivamente $(9,23)$.

Tiene actividad contra parásitos internos (nemátodos) y parásitos externos (artrópodos) de caninos, ovinos, caprinos, equinos, bovinos, ciervos rojos, porcinos y venados $(1,3)$.

En numerosas pruebas de campo ha demostrado ser efectiva contra estadíos inmaduros y adultos de Ostertagia ostertagi, Dictyocaulus viviparus, Haemonchus contortus, H. placei, Trichostrongylus axei, Cooperia oncophora. Así mismo, tiene acción contra garrapatas del género Boophilus, ácaros de la sarna y piojos (1).

\section{SEGURIDAD.}

Las reacciones adversas de la moxidectina en ovinos ocurren cuando se aplican de dos a cinco veces la dosis recomendada $(0.4$ a $1.0 \mathrm{mg} / \mathrm{kg}$ p.v. $)$; sin embargo, no existen reacciones adversas con tratamientos sucesivos (dos veces) a la dosis terapéutica. No ocurre muerte de bovinos cuando son inyectados subcutáneamente hasta 10 veces la dosis recomendada $(2.0 \mathrm{mg} / \mathrm{kg}$ p.v). A pesar de esto, se debe tener cuidado especial en la dosificación de los animales menores de $100 \mathrm{~kg}$. de peso vivo, porque podrían ser susceptibles a la sobredosis (3). En perros collies, Allan y col. (10) encontraron que la moxidectina a dosis de 0.03 , 0.06 y $0.09 \mathrm{mg} / \mathrm{kg}$ p.v. no producen signos de toxicidad.

El fármaco es seguro en animales en etapa de reproducción. Cuando fue aplicado tres veces la dosis recomendada, no se observó efectos sobre la reproducción de toros y vacas gestantes. Tres veces la dosis recomendada $(0.6 \mathrm{mg} / \mathrm{kg}$ p.v $)$ no tuvo efecto en vacas y vaquillas cuando se inyectaron en cada trimestre de la gestación (11).

\section{PRESENTACIÓN Y DOSIFICACIÓN.}

Las presentaciones de este producto son en suspensión oral, inyectable y "pour-on". La suspensión oral e inyectable son formulaciones acuosas, que contienen propilenglicol y solubilizantes; mientras que la forma "pour-on" tiene únicamente un vehículo (1).

La dosis para la vía oral e inyectable es de $0.2 \mathrm{mg} / \mathrm{kg}$ p.v. y la forma "pour-on" es utilizada a una dosis de $0.5 \mathrm{mg} / \mathrm{kg}$ p.v. En Japón es utilizada la presentación en tabletas a dosis de $0.03 \mathrm{mg} /$ $\mathrm{kg}$, administradas mensualmente para prevenir Dirofilaria immitis en perros (1).

La fórmula oral e inyectable, administrada a una dosis de $0.2 \mathrm{mg} / \mathrm{kg}$ de p.v. es efectiva contra infecciones naturales y artificiales de nemátodos gastrointestinales en vacas y ovejas (12-14).

\section{PERSISTENCIA.}

En estudios realizados por Kerboeuf y col. (15) y Dorchies y col. (16) demostraron que en ovinos, las formulaciones oral y subcutánea presentaron una efectividad del $100 \%$ contra $T$. circumcincta y $H$. contortus por más de 35 días después del tratamiento.

Abboutt y col. (17), reportaron un efecto de persistencia superior al 99\% cuando usaron la moxidectina por la vía oral contra $H$. contortus por más de 28 días.

La eficacia de la moxidectina inyectable al $1 \%$ contra T. columbriformis es mejor (99\%) que la suspensión oral y la efectividad persiste por más de 21 días después del tratamiento; además reduce moderadamente la carga parasitaria por más de 35 días (17).

En vacas, diversos estudios demostraron que la efectividad (cerca del 100\%) de la moxidectina contra $O$. ostertagi persistió al menos cinco semanas, cuando se utilizó en solución inyectable, y seis semanas para la formulación "pour-on." La persistencia para $D$. viviparus es de al menos seis semanas para la presentación "pour-on" e inyectable (18-21). La moxidectina inyectable al $1 \%$ es menos efectiva contra $C$. oncophora, pero reduce la postura de huevos en un $95 \%$ durante dos a tres semanas (22). 
G Aguilar-Tipacamú, RI Rodríguez-Vivas.

\section{EFECTIVIDAD DE LA MOXIDECTINA PARA EL TRATAMIENTO DE LOS PARÁSITOS EN LOS ANIMALES.}

\section{OVINOS Y CAPRINOS.}

\section{Endoparásitos.}

En ovinos, la administración oral de la moxidectina presenta una eficacia mayor al $99 \%$ contra larvas y estados adultos de nemátodos del género Haemonchus, Ostertagia, Trichostrongylus, Cooperia, Oesophagostomun, Chabertia y Dictyocaulus. También se ha obtenido buen control hacia Psoregates ovis (1).

La moxidectina inyectable es menos efectiva que la suspensión oral contra larvas del género Nematodirus y tiene bajo efecto contra las formas adultas de este género (23).

La moxidectina actualmente no se encuentra en el mercado para ser utilizada en cabras $(24,25)$ y por consiguiente se desconoce la dosis recomendada para esta especie. Sin embargo, hay evidencias que sugieren que a dosis de $0.2 \mathrm{mg} / \mathrm{kg}$ de peso corporal, puede ser efectiva para reducir el conteo de huevos en cabras naturalmente infectadas (23, 26-28).

Se han realizado diversos estudios para comprobar la eficacia de la moxidectina contra endoparásitos del ganado ovino, dentro de estos estudios se encuentra el realizado por Pankavich y col. (29), donde se evaluaron la eficacia de la ivermectina, nemadectina y moxidectina por vía oral en corderos infectados con una cepa sensible de $H$. contortus y con una cepa resistente a la ivermectina. Los corderos fueron tratados por vía oral a una dosis de $0.2 \mathrm{mg} / \mathrm{kg}$ p.v. Se observó que la ivermectina no redujo significativamente el número de huevecillos y adultos de $H$. contortus resistentes a la ivermectina, no siendo así para la nemadectina y moxidectina que presentaron una efectividad del $99 \%$ y $100 \%$ respectivamente. Los tres antihelmínticos fueron efectivos (100\%) contra la cepa sensible. Además encontraron que la cepa resistente empezó a producir huevos a los 20-21 días, mientras que la cepa sensible lo hizo producir a los 15-16 días.

Otro experimento realizado por Craig y col. (30), mostraron la efectividad de la moxidectina e ivermectina en ovejas contra cepas de $H$. contortus resistentes a la ivermectina. La moxidectina demostró ser efectiva a dosis de 0.2 y $0.4 \mathrm{mg} / \mathrm{kg}$ p.v. con $99.9 \%$ y $100 \%$, respectivamente hacia las cepas resistentes. Ambos antihelmínticos fueron efectivos contra la cepa sensible de $H$. contortus, presentando la moxidectina una eficacia del $100 \%$ y la ivermectina del $99.7 \%$. La ivermectina tuvo una eficacia del $38.8 \%$ y $53.1 \%$ en las cepas resistentes tratados con 0.4 y $0.8 \mathrm{mg} / \mathrm{kg}$ p.v. respectivamente.

Kerboeuf y col. (15), reportaron una efectividad de la moxidectina oral y subcutánea del 100\% contra parásitos ( $T$. circumcincta y $\mathrm{H}$. contortus) por más de 35 días, utilizando una dosis de $0.2 \mathrm{mg} / \mathrm{kg}$ p.v. También administraron moxidectina a ovejas días antes de la infección artificial con larvas de Teladorsagia, Haemonchus y Trichostrongylus. La eficacia de la moxidectina inyectable contra T. columbriformis fue excelente (99\%) cuando fue administrada a los 14 y 21 días antes de la infección. La efectividad de la suspensión oral fue del $100 \%$ por un período de 28 días contra T. circumcincta y H. contortus. En animales tratados 35 días antes de la infección la eficacia fue del $100 \%$ contra $H$. contortus y más del 99\% contra T. circumcincta.

Otro estudio realizado por Uriarte y col. (31), encontraron una eficacia de la moxidectina en suspensión oral del $100 \%$ contra larvas $\mathrm{L}_{4}$ de $H$. contortus, $O$. circumcincta, $T$. columbriformis y T. vitrinus, a las cuatro semanas post-infección a una dosis de $0.2 \mathrm{mg} / \mathrm{kg}$ p.v.. La eficacia para los estados adultos fue del $100 \%$ para $H$. contortus, T. vitrinus y T. colubriformis, y del $99.8 \%$ para O. circumcincta.

\section{Ectoparásitos.}

La moxidectina es eficaz para el control de ectoparásitos en ovinos; Fthenakis y col. (32), demostraron la eficacia de la moxidectina contra

\section{Revista Biomédica}


Tratamiento con moxidectina en animales.

la sarna sarcóptica.

Cuando se aplicó una sola dosis de $0.2 \mathrm{mg} / \mathrm{kg}$ p.v. por vía subcutánea, se redujo el porcentaje de infección por Sarcoptes scabiei de un $75 \%$ a un $92 \%$. Aplicando dos dosis de $0.2 \mathrm{mg} / \mathrm{kg}$ cada 10 días, las lesiones en la piel desaparecieron en un $100 \%$. En este estudio las ovejas tratadas produjeron $22.4 \%$ más leche que las que estaban infestadas, y los corderos tuvieron ganancias del $19.4 \%$ más de peso que los infestados.

Corba y col. (33) encontraron que la moxidectina subcutánea tuvo un efecto del $100 \%$ para el control de Psoroptes ovis y S. sabie (variedad ovis) en borregas tratadas $(0.2 \mathrm{mg} / \mathrm{kg}$ p.v.) dos veces con intervalo de 7 días.

\section{BOVINOS.}

\section{Endoparásitos.}

Adams (1), reportó que en rumiantes, principalmente en bovinos, la moxidectina (inyectable o "pour-on") tiene alta eficacia, siendo mayor al 99\% contra larvas hipobióticas y estadíos adultos de $O$. ostertagi, estados adultos de $H$. placei, T. axei, T. columbriformis, T. discolor, $O$. radiatum y Bonustomun phlebotomun. Es menos eficaz contra Nematodirus helvetianus (95\%) y efectiva en un rango del 92-100\% contra estadíos adultos de $C$. oncophora y larvas de $C$. punctata, larvas y estados adultos de $C$. pectinata y $C$. spatulata.

En estudios realizados por diversos autores, reportan la efectividad de la moxidectina contra diversos endoparásitos de los bovinos (cuadro 1).

\section{Ectoparásitos.}

La población de garrapatas $B$. microplus es reducida en más del $95 \%$ en bovinos seguida de una dosis de moxidectina vía subcutánea, suprimiéndose hasta por 32 días. También la moxidectina inyectable es eficiente para el control de piojos chupadores (99-100\%), contra Haematopinus euryternus, Linognathus vituli y Solenoptes capillatus y miasis bovina (Hypoderma lineatum). Sin embargo, la formulación "pour-on" proporciona mejor control de piojos mordedores (Damalinia bovis) que la formulación inyectable (37). Lonneux y Losson (36) demostraron que la moxidectina "pour on" $(0.5 \mathrm{mg} / \mathrm{kg}$ p.v. $)$ tiene una efectividad del $100 \%$ contra $H$. bovis.

Estudios realizados en ectoparásitos del ganado bovino demuestran eficacias que van del 95 al $100 \%$ (cuadro 2).

\section{EQUINOS.}

En equinos la moxidectina en gel es efectiva en un $99 \%$ contra los estadíos adultos y larvarios de Habronema muscae, Parascaris equorum y Oxuris equi utilizando una dosis de $0.3 \mathrm{mg} / \mathrm{kg}$ p.v. (1).

En un estudio realizado por Monahan y col. (41), evaluaron la moxidectina oral contra parásitos gastrointestinales utilizando dosis de $0.3,0.4$ y 0.5 $\mathrm{mg} / \mathrm{kg}$ p.v., encontrando una efectividad en todas las dosis del $100 \%$ hacia estadíos adultos de Strongylus vulgaris, S. edentatus, Triodontophorus ssp. y 22 especies de estrongílidos del intestino delgado de los equinos. La moxidectina también fue efectiva al $100 \%$ contra larvas de S. edentatus y O. equi. Al mismo tiempo presentó una efectividad del $94 \%$ contra adultos de O. equi y larvas de $S$. vulgaris.

La moxidectina tuvo una efectividad del $89 \%$ contra Gasterophilus intestinalis a una dosis de $0.3 \mathrm{mg} / \mathrm{kg}$ p.v. y $96 \%$ de efectividad a una dosis de 0.4 y $0.5 \mathrm{mg} / \mathrm{kg}$ p.v. (41).

Xiao y col. (42), encontraron que la moxidectina en equinos a una dosis de 0.3 y 0.4 $\mathrm{mg} / \mathrm{kg}$ p.v. reducen en un $99.8 \%$ los estrongílidos después de 14 días de aplicado el tratamiento. La moxidectina a una dosis de $0.3 \mathrm{mg} / \mathrm{kg}$ p.v. presentó una efectividad del $88.2 \%$ contra adultos de $O$. equi. y a una dosis de $0.4 \mathrm{mg} / \mathrm{kg}$ p.v. presentó una eficacia del $100 \%$ contra adultos de $O$. equi, $H$. muscae, T. tenuicollis, T. brevicauda, T. serratus, $S$. vulgaris, $S$. edentatus y larvas $\mathrm{L}_{4}$ de $O$. equi.

Lyons y col. (43), probaron la efectividad de la moxidectina contra infecciones naturales de parásitos internos de equinos y encontraron que a 
G Aguilar-Tipacamú, RI Rodríguez-Vivas.

\section{Cuadro 1}

Efecto de la moxidectina contra parásitos internos de los bovinos, según varios tores.

\begin{tabular}{|c|c|c|c|c|}
\hline Dosis & Vía & Parásitos & Efectividad & Autor \\
\hline $0.5 \mathrm{mg} / \mathrm{kg}$ p.v. & "pour-on" & $\begin{array}{l}\text { Cooperia spp. } \\
\text { O. radiatum } \\
\text { Trichostrongylus spp. } \\
\text { O. ostertagi }\end{array}$ & $\begin{array}{l}94.4-100 \% \\
100 \% \\
100 \% \\
100 \%\end{array}$ & Yazwinski y col. (34) \\
\hline $0.2 \mathrm{mg} / \mathrm{kg} \mathrm{p.v}$ & Subcutánea & D. viviparus & $86 \%$ & Hong y col. (35) \\
\hline $0.2 \mathrm{mg} / \mathrm{kg}$ p.v. & Subcutánea & $\begin{array}{l}\text { D. viviparus } \\
\text { B. phlebotomun } \\
\text { O. ostertagi } \\
\text { O. lyrata } \\
\text { H. placei } \\
\text { T. axei } \\
\text { C. punctata } \\
\text { C. spatulata } \\
\text { C. pectinata } \\
\text { O. radiatum } \\
\text { T. discolor }\end{array}$ & $100 \% *$ & Williams y col. (13) \\
\hline $\begin{array}{l}0.2 \mathrm{mg} / \mathrm{kg} \text { p.v. } \\
0.3 \mathrm{mg} / \mathrm{kg} \text { p.v. }\end{array}$ & Subcutánea & $\begin{array}{l}\text { Ostertagia spp. } \\
\text { Trichostrongylus spp. } \\
\text { Nematodirus spp. } \\
\text { Trichuris spp. } \\
\text { Oesophagostomun spp. } \\
\text { Dictyocaulus spp. }\end{array}$ & $99 \%$ & Ranjan y col. (12) \\
\hline $0.2 \mathrm{mg} / \mathrm{kg}$ p.v. & Subcutánea & C. oncophora & $94 \%$ & Ranjan y col. (12) \\
\hline $0.3 \mathrm{mg} / \mathrm{kg}$ p.v. & Subcutánea & Cooperia spp. & $98 \%$ & Ranjan y col. (12) \\
\hline
\end{tabular}

* Tiene efectividad contra larvas $\mathrm{L}_{4}$.

dosis de $0.2 \mathrm{mg} / \mathrm{kg}$ p.v. por vía subcutánea se larvas $\mathrm{L}_{4} G$. nasalis, ascáridos y $S$. vulgaris. eliminaron en un $89 \%$ las larvas $\mathrm{L}_{2}$ de $G$. intestinalis Utilizando $0.4 \mathrm{mg} / \mathrm{kg}$ p.v. se eliminaron del 93 al y un $88 \%$ las larvas $\mathrm{L}_{3}$; fue $100 \%$ efectiva contra $100 \%$ de larvas $\mathrm{L}_{2}$ de $G$. intestinalis y del 89 al

Cuadro 2

Efecto de la moxidectina contra parásitos externos de los bovinos, según varios autores.

\begin{tabular}{lllll}
\hline Dosis & Vía & Parásitos & Efectividad & Autor \\
\hline $0.2 \mathrm{mg} / \mathrm{kg}$ p.v. & Subcutánea & $\begin{array}{l}\text { Psoroptes ovis } \\
\text { Psoroptes ovis }\end{array}$ & $100 \%$ & $100 \%$ \\
$0.5 \mathrm{mg} / \mathrm{kg}$ p.v. & "pour-on" & & Lonneux y Losson (38) \\
& & & \\
$0.2 \mathrm{mg} / \mathrm{kg}$ p.v. & Subcutánea & B. microplus & $99-100 \%$ & Remington y col. (39) \\
$0.2 \mathrm{mg} / \mathrm{kg}$ p.v. & Subcutánea & B. microplus & $97-99 \%$ & Carascostantogolo y col. (4) \\
$0.5 \mathrm{mg} / \mathrm{kg}$ p.v. & "pour-on" & B. microplus & $95 \%$ & Guglielmone y col. (40) \\
\hline
\end{tabular}

\section{Revista Biomédica}


Tratamiento con moxidectina en animales.

Cuadro 3

Efecto de la moxidectina contra parásitos internos de los cerdos, según Stewart y col. (46).

\begin{tabular}{llc}
\hline Dosis & \multicolumn{1}{c}{ Parásitos } & Efectividad \\
\hline $1.25 \mathrm{mg} / \mathrm{kg}$ p.v. & Ascaris suum & $98.3 \%$ \\
$0.75,1.00,1.25$ y $1.50 \mathrm{mg} / \mathrm{kg}$ p.v. & Metastrongylus spp. & $100 \%$ \\
$1.50 \mathrm{mg} / \mathrm{kg}$ p.v. & O. quadrispinulatum & $100 \%$ \\
$0.75 \mathrm{mg} / \mathrm{kg}$ p.v. & T. suis & $93.5 \%$ \\
\hline
\end{tabular}

$100 \%$ de larvas $\mathrm{L}_{3}$; además a la misma dosis fue efectiva al $100 \%$ contra larvas $\mathrm{L}_{3}$ de $G$. nasalis, adultos de $P$. equorum, $O$. equi, $S$. vulgaris y $S$. edentatus.

Reinemeyer y col. (44) encontraron que la moxidectina a una dosis de $0.4 \mathrm{mg} / \mathrm{kg}$ p.v. por vía oral (gel) tuvo una efectividad del $100 \%$ contra adultos de $G$. nasalis y $G$. intestinalis, y una efectividad del $100 \%$ y $97.6 \%$ contra larvas $\mathrm{L}_{3}$ de G. nasalis y $G$. intestinalis respectivamente.

\section{OTRAS ESPECIES.}

La moxidectina es $100 \%$ efectiva contra larvas de Dirofilaria immitis al utilizar dosis de $0.3 \mathrm{mg} /$ $\mathrm{kg}$ de p.v. (1). Además es altamente efectiva contra Ancylostoma caninum.

En ciervos rojos, la eficacia de la moxidectina "pour-on" es del 100\% contra estadíos adultos e inmaduros de D. viviparus. En venados fue efectiva en un 99.7-100\% contra estadíos adultos e inmaduros de parásitos pulmonares. En ambos animales es efectiva en un $99.9 \%$ contra estadíos adultos y larvas de Ostertagia spp. (45).

Stewart y col. (46), realizaron un experimento utilizando un tratamiento "pour-on" de moxidectina en cerdos (cuadro 3).

\section{CONCLUSIONES.}

Se concluye que la moxidectina es altamente efectiva para el tratamiento de parásitos internos y artrópodos de los animales. Su efectividad se ha probado principalmente en bovinos, ovinos y equinos.

\section{REFERENCIAS.}

1.- Adams R. Veterinary Pharmacology and Therapeutics.

$7^{a}$ ed. Des Moines: Iowa State University Press/ Ames; 1995. p. 923-25.

2.- Sumano LH, Ocampo CL. Farmacología Veterinaria. 2da. Ed. México: 1997. p. 282-3.

3.- Courtney CH, Roberson EL. Chemotherapy of parasitic diseases. In: Adams R. Editor. Veterinary Pharmacology and Therapeutics. $7^{\mathrm{a}}$ edición. Des Moines: Iowa State University Press/Ames; 1995. p. 923-5.

4.- Carascostantogolo J, Eddi CS,Bulman GM, Morley ME, Noaco A. Proc. $14^{\text {th }}$ Ibt. Conf. WAAVP, Cambridge; 1993. p. 201.

5.- Sibson GJ. The effects of moxidectin against natural infestation of cattle tick (Boophilus microplus). Aust Vet J 1994; 71: 22-3.

6.- Booth NH, McDonald LE. Farmacología y Terapéutica Veterinaria. Volumen II. Zaragoza: Acribia; 1987. p. 1779.

7.- Mudd AJ, Parker LD. Use of the new molecule moxidectin for the control of parasites in sheep. Proceedings of the Sheep Veterinary Society 1993-1994 1995; 18: 139143.

8.- Gill JH, Lacey E. Avermectin/milbemycin resistence in Trichostrongyloid nematodes. Int J Parasitol 1998; 28: 86377.

9.- Escudero E, Carceles CM, Díaz MS, Sutra JF, Galtier $\mathrm{P}$, Alvinerie M. Pharmacokinetics of moxidectin and doramectin in goats. Res Vet Sci 1999; 67: 177-81.

10.- Allan JP, Tranquilli WJ, Hutchens DE. Safety of moxidectin in avermectin-sensitive Collies. Am J Vet Resl 2000; 61: 482-3. 


\section{G Aguilar-Tipacamú, RI Rodríguez-Vivas.}

11.- Rae DO, Larsen RE, Wang GT. Safety assement of moxidectin $1 \%$ injectable on reproductive performance in beef cows. Am J Vet Res 1994; 55: 251-3.

12.- Ranjan S, Trudeau C, Princhard RK, Von Kutzleben $\mathrm{R}$, Carrier D. Efficacy of moxidectin against naturally acquired nematode infections in cattle. Vet Parasitol 1992; 41: $227-31$.

13.- William JC, Nault C, Ramsey RT, Wang GT. Efficacy of Cydectin moxidectin 1\% injectable aganist experimental infections of Dictyocaulus viviparus and Bunostomun phlebotomum superimposed on natural gastrointestinal infections in calves. Vet Parasitol 1992; 43: 293-9.

14.- Zimmerman GL, Hoberg EP, Pankavich JA.. Efficacy of orally administered moxidectin against naturally acquired gastrointestinal nematodes in cattle. Am J Vet Res 1992; 53: 1409-10.

15.- Kerboeuf D, Hubert J, Cardinaud B, Blond F. The persistence of the efficacy of injectable or oral moxidectin against Teladorsagia, Haemonchus and Trichostrongylus species in experimentally infected sheep. Vet Record 1995; 137: 399-401.

16.- Dorchies P, Cardinaud B, Fournier R. Efficacy of moxidectin as a $1 \%$ injectable and a $0.1 \%$ oral drench against nasal bots, pulmonary and gastrointestinal nematodes in sheep. Vet Parasitol 1996; 65: 163-8.

17.- Abboutt KA, Cobb RM, Holm Glass M. Duration of the activity of moxidectin against Haemonchus contortus in sheep. Aust Vet J 1995; 72: 408-10.

18.- Eysker M, Eilers C. Persistence of the effect of a moxidectin pour-on against naturally acquired cattle nematodes. Vet Record 1995; 137: 457-60.

19.- Hubert J, Kerboeuf D, Cardinaud B, Blond F. Persistent efficacy of moxidectin against Dictyocaulus viviparus and Ostertagia ostertagi in cattle. Vet Record 1995 ; 136: 223 4.

20.- Hubert J, Kerboeuf D, Cardinaud B, Blond F, Fournier R. Persistent efficacy of topical moxidectin against Dictyocaulus viviparus and Ostertagia ostertagi in cattle. Vet Parasitol 1997; 68: 187-90.

21.- Vercruysse J, Claerebout E, Dorny P, Demeulenare D, Deroover E. Persistence of the efficacy of pour-on and injectable moxidectin against Ostertagia ostertagi and Dictyocaulus viviparus in experimentally infected cattle.
Vet Record 1997; 140: 64-6.

22.- Eysker M, Boersema JH, Cornelissen JB, Kloosterman A, Kooyman FNJ. Residual effect of injectable moxidectin against lungworm and gastrointestinal nematodes incalves exposed to high pasture infectivity levels in the Netherlands. Vet Parasitol 1996; 61: 61-71.

23.- Torres AJF. Suplementary feeding and the control of gastrointestinal nematodes of goats in Yucatán, México. $\mathrm{PhD}$ thesis. University of London; 1999. p. 1-269.

24.- Bailey KM. Anthelmintic use in goats and the route of administration. New Zealand Vet J 1994; 42: 37-8.

25.- Cobb RM, Murphy AW. Use of moxidectin in goats in New Zealand. Vet Record 1995; 137: 496.

26.- Pomroy WE, Whelan N, Alexander AM, West DW, Stafford K, Adlington BA, Calder SM. Multiple resistence in goat-derived Ostertagia and the efficacy of moxidectin and combinations of other anthelmintics. New Zealand Vet J 1992; 40: 76-8.

27.- Praslicka J, Várady M, Corba J. Persistent infection with multiple anthelmintic resistant gastrointestinal nematodes in Cashmere goats. Vet Res Communications 1994; 18: 443-6.

28.- Leathwick DM. A case of moxidectin failing to control ivermectin resistant Ostertagia species in goats. Vet Record 1995; 136: 443-4.

29.- Pankavich JA, Berger H, Simkins K. Efficacy of moxidectin, nemadectin and ivermectin against an ivermectin-resistant strain of Haemonchus contortus in sheep. Vet Record 1992; 130: 241-3.

30.- Craig TM, Hatfield TA, Pankavich JA, Wang GT. Efficacy of moxidectin against an ivermectin resistant strain of Haemonchus contortus in sheep. Vet Parasitol 1992; 41: 329-33.

31.- Uriarte J, Gracia MJ, Almeria S. Efficacy of moxidectin against gastrointestinal nematode infections in sheep. Vet Parasitol 1994; 51: 301-5.

32.- Fthenakis GC, Papadopoulos E, Himonas C, Leontides L. Efficacy of moxidectin against sarcoptic mange and effects on milk yield of ewes and growth of lambs. Vet Parasitol 2000; 87: 207-16.

33.- Corba J, Varady M, Praslicka J, Tomasovicova O. 
Tratamiento con moxidectina en animales.

Efficacy of injectable moxidectin against mixed (Psoroptes ovis and Sarcoptes scabiei var. Ovis) mange infestation in sheep. Vet Parasitol 1995; 56: 339-44.

34.- Yazwinski TA, Tucker C, Copeland S, Yazwinski T, Guerino F. Dose confirmation of moxidectin pour-on against natural nematode infections in lactating dairy cows. Vet Parasitol 1999; 86: 223-8.

35.- Hong C, Harris TJ, Grimshow TR, Newcomb KM. Persistent activity of ivermectin topical and moxidectin injection against Ostertagia ostertagi and Dictyocaulus viviparus in calves. Vet Record 1995; 137: 640-1.

36.- Lonneux JF, Losson BJ. The efficacy of moxidectin $0.5 \%$ pour-on against Hypoderma bovis in naturally infested cattle: parasitological and serological data. Vet Parasitol 1994; 52: 313-20.

37.- Chick KB, McDonald D, Cobb R, Kieran PJ, Wood I. The efficacy of inyectable and pour-on formulations of moxidectin against lice on cattle. Aust Vet J 1993; 70: 212-4.

38.- Lonneux JF, Losson B. Field efficacy of injectable and pour-on moxidectin in cattle naturally infested with Psoroptes ovis (Acarina:Psoroptidae). Vet Parasitol 1992; 45:147-52.

39.- Remington B, Kieran P, Coob R, Bodero D. The aplication of moxidectin formulations for control of the cattle tick (Boophilus microplus) under Queensland field conditions. Aust Vet J 1997; 75: 588-91.

40.- Guglielmone AA, Mangold AJ, Muñoz CM, Scherling N, García RF, Anziani OS, Loppolo M. Moxidectin pouron for control of natural populations of the cattle tick Boophilus microplus (Acarina: Ixodidae). Vet Parasitol 2000; 87: 237-41.

41.- Monahan CM, Chapman MR, French DD, Taylor HW, Klei TR. Dose titration of moxidectin oral gel against gastrointestinal parasites of ponies. Vet Parasitol 1995; 59: 241-8.

42.- Xiao L, Herd RP, Majewski GA. Comparative efficacy of moxidectin and ivermectin against hypobiotic and encysted cyathostomes and other equine parasites. Vet Parasitol 1994; 53: 83-90.

43.- Lyons ET, Tolliver SC, Drudge JH, Granstrom DE, Collins SS, Stamper S. Critical and controlled tests of activity of moxidectin (CL 301,423) against natural infections of internal parasites of equids. Vet Parasitol 1992; 41: $255-84$.

44.- Reinemeyer CR, Scholl PJ, Andrews FM, Rock DW. Efficacy of moxidectin equine oral gel against endoscopically confirmed Gasterophilus nasalis y Gasterophilus intestinallis (Diptera: Oestridae) infections in horses. Vet Parasitol 2000; 88: 287-91.

45.- Waldrup KA, Mackintosh CG, Duffy MS, Labes RE, Johnstone PD, Taylor MJ, Murphy AW. The efficacy of a pour-on formulation of moxidectin in young red and wapití hybrid deer. New Zealand Vet J 1998; 46: 182-5.

46.- Stewart TB, Wiles SE, Miller JE, Rulli RD. Efficacy of moxidectin $0.5 \%$ pour-on against swine nematodes. Vet Parasitol 1999; 87: 39-44. 